\title{
SYMMETRIC SEMICONTINUITY IMPLIES CONTINUITY BY
}

JAROMIR UHER

\begin{abstract}
The main result of this paper shows that, for any function, symmetric semicontinuity on a measurable set $E$ implies continuity a.e. in $E$ and, similarly, that symmetric semicontinuity on a set residual in $R$ implies continuity on a set residual in $R$. These propositions are used to prove more precise versions of the fundamental connections between symmetric and ordinary differentiability.
\end{abstract}

In this note we employ the main idea from [9] to study the problem of measurability of functions with some "regular" symmetric behaviour. Our method leads immediately to a proof of continuity or differentiability properties of such functions.

The fundamental connections between symmetric and ordinary differentiability and between symmetric and ordinary continuity for measurable functions have been established by Khintchine [4] and Stein and Zygmund [8]. To prove similar results for general functions seemed to be a much harder task. Charzynski [2] has shown that a finitely symmetrically differentiable function is differentiable a.e., Fried [3] and Preiss [6] proved that any symmetrically continuous function is continuous on a residual set and on a set of full measure, respectively; and Belna [1] proved that a function symmetrically continuous on a measurable set $E$ is continuous a.e. in $E$. Finally, Uher [9] in a rather complicated paper showed that functions possessing a symmetric derivative (possibly infinite) a.e. are differentiable a.e.

The main idea of [9] was to use symmetric semicontinuity, which follows from the existence of the symmetric derivative, while symmetric continuity does not. Here we shall see that the strange mixture of differentiability and continuity properties in [9] is not necessary. Namely, we show that symmetric semicontinuity a.e. already implies continuity a.e. (See Theorem 1, which gives a considerably more general result.) A similar result is also proved with sets of measure zero replaced by sets of the first category. (See Theorem 1 again.) This basic connection between symmetric and ordinary properties is then used to prove more precise versions of the results mentioned above.

Recall that the upper symmetric derivative of a function $f$ at $x \in R$ is

$$
\bar{f}^{s}(x)=\limsup _{h \rightarrow 0} \frac{f(x+h)-f(x-h)}{2 h}
$$

and that the lower symmetric derivative is

$$
\underline{f}^{s}(x)=\liminf _{h \rightarrow 0} \frac{f(x+h)-f(x-h)}{2 h} .
$$

Received by the editors April 10, 1985.

1980 Mathematics Subject Classification. Primary 26A15; Secondary 26A24. 
If $\bar{f}^{s}(x)=f^{s}(x)$, the common value, finite or infinite, is called the symmetric derivative of $f$ at $x$. A function $f$ defined on $R$ is said to be upper (lower) symmetrically semicontinuous at $x$ if

$$
\limsup _{h \rightarrow 0_{+}} f(x+h)-f(x-h) \leqslant 0 \quad\left(\liminf _{h \rightarrow 0_{+}} f(x+h)-f(x-h) \geqslant 0\right) .
$$

It is said to be symmetrically semicontinuous at $x$ if it is upper or lower symmetrically semicontinuous at $x$. We shall also use the usual notations $\bar{D} f(x), \underline{D} f(x)$, $\bar{D}^{+} f(x), \bar{D}^{-} f(x), \underline{D}^{+} f(x)$, and $\underline{D}^{-} f(x)$ for ordinary, one-sided, upper, and lower derivatives. If $M$ is a subset of the real line, we denote by $|M|$ its outer Lebesgue measure,

$$
D(M)=\left\{x \in R ; \lim _{h \rightarrow 0_{+}} \frac{|M \cap(x-h, x+h)|}{2 h}=1\right\},
$$

and, finally, $a(M)$ is the union of all open intervals intersecting $M$ in a set of the first category.

Since we do not want to repeat practically the same proofs in the case of sets of Lebesgue measure zero and in the case of sets of the first category, we shall define families of exceptional sets fulfilling certain properties. Hence, in the following text, $\mathscr{T}$ will be a family of subsets of $R$ given by $\mathscr{T}=\{A \subset R ; b(A)=\varnothing\}$, where $b$ is a map associating with each set $A \subset R$ a set $b(A) \subset R$ satisfying the following conditions.

(i) If $A \subset B$, then $b(A) \subset b(B)$.

(ii) If $\bigcup_{n=1}^{\infty} A_{n}=A$, then $b(A) \backslash \bigcup_{n=1}^{\infty} b\left(A_{n}\right) \in \mathscr{T}$.

(iii) If $A \backslash B \in \mathscr{T}$ and $B \backslash A \in \mathscr{T}$, then $b(A)=b(B)$.

(iv) $b(b(A))=b(A)$ for each $A \subset R$.

(v) $A \backslash b(A) \in \mathscr{T}$ for each $A \subset R$.

(vi) $b(\alpha+\beta A)=\alpha+\beta b(A)$ for each $A \subset R$ and $\alpha, \beta \in R$.

For convenience, we shall call a set $A \subset R$ measurable if $b(A) \backslash A \in \mathscr{T}$. We shall also assume that the following three conditions hold.

(vii) Each open set $A \subset R$ is measurable.

(viii) If $A$ is measurable, then $b(A) \cap b(B)=b(A \cap B)$ for every $B \subset R$.

(ix) If at least $(k-1)$ sets among $A_{1}, \ldots, A_{k}$ are measurable and if $0 \in b\left(A_{i}\right)$ for each $i=1, \ldots, k$, then for every $p>0$ there is $\Delta>0$ with the following property:

Whenever $\delta \in(0, \Delta), I \subset(-\delta, \delta)$ is an open interval with length at least $\delta / p$ and whenever $\left|\alpha_{i}\right| \leqslant \delta$ for $i=1, \ldots, k$, then

$$
I \cap \bigcap_{i=1}^{k}\left(\alpha_{i}+A_{i}\right) \notin \mathscr{T} .
$$

Remark 1. (a) From conditions (i), (ii) and (iv) we easily see that $\mathscr{T}$ is a $\sigma$-ideal. (Recall that a set $\mathscr{T}$ of subsets of $R$ is called a $\sigma$-ideal if $\left\{A_{n}\right\}_{n=1}^{\infty} \subset \mathscr{T}$ implies $\cup_{n=1}^{\infty} A_{n} \in \mathscr{T}$ and if $A \in \mathscr{T}$ and $B \subset A$ then $B \in \mathscr{T}$.) Moreover, $\varnothing \in \mathscr{T}$, since (ii) implies that $\mathscr{T}$ is nonempty. 
(b) Measurable sets form a $\sigma$-algebra of subsets of $R$. Since $R$ is measurable according to (vii) and since (ii) implies that a countable union of measurable sets is measurable, it suffices to verify that the complement of a measurable set is again measurable. But this follows from (viii) and (v), since for any measurable set $A$ we have

$$
\begin{aligned}
b(R \backslash A) \backslash(R \backslash A) & =b(R \backslash A) \cap A \subset(b(R \backslash A) \cap b(A)) \cup(A \backslash b(A)) \\
& =b((R \backslash A) \cap A) \cup(A \backslash b(A))=A \backslash b(A) .
\end{aligned}
$$

(c) Using condition (iv), we immediately see that for each $A \subset R$ the set $b(A)$ is measurable.

(d) From (vi), (ix) and from the measurability of one-point sets we deduce that one-point sets belong to $\mathscr{T}$; hence $\mathscr{T}$ contains all countable sets.

(e) Putting $p \geqslant 1$ in (ix) we see that $(0, \delta) \cap \cap_{i=1}^{k}\left(\alpha_{i}+A_{i}\right) \notin \mathscr{T}$ and $(-\delta, 0) \cap$ $\bigcap_{i=1}^{k}\left(\alpha_{i}+A_{i}\right) \notin \mathscr{T}$.

(f) As usual, whenever $P$ is any property of points in $R$, we shall say that $P$ holds almost everywhere in $A$ (or at almost every point of $A$ ) if there is a set $B \in \mathscr{T}$ such that $P$ holds at every point of $A \backslash B$.

If we want to study sets of measure zero, we define $b(A)$ as the set of all outer density points of $A$. Then $\mathscr{T}$ is precisely the $\sigma$-ideal of sets of Lebesgue measure zero and the measurable sets coincide with the Lebesgue measurable sets. The properties (i)-(viii) are well known and condition (ix) follows easily from the definition of the density and of the outer density.

If we want to study sets of the first category, we define $b(A)=R \backslash$ Closure $(a(A))$. Now $\mathscr{T}$ is the $\sigma$-ideal of sets of the first category and the measurable sets are precisely the sets with the Baire property. Proving (i)-(viii) is easy and to prove (ix), it suffices to choose $\delta>0$ (independent of $p$ ) such that $(-2 \delta, 2 \delta) \subset b\left(A_{i}\right)$ for each $i=1, \ldots, k$.

Lemma 1. Assume that $f$ is a real-valued function defined on the real line, $\varepsilon$ and $\eta$ are positive numbers and $E \subset R$. Assume further that $f(t+h)-f(t-h)>-\varepsilon$ for every $t \in E$ and $h \in(0, \eta)$ and that $f$ is symmetrically semicontinuous at a.e. point of $b(E)$. Then $\liminf x_{x \rightarrow z_{+}} f(x) \geqslant f(z)-5 \varepsilon$ for each $z \in b(E)$.

Proof. Considering, if necessary, the function $\hat{f}(t)=\frac{1}{\varepsilon} f(t / \eta)$, we see that it suffices to assume that $\varepsilon=\eta=1$. Moreover, it also suffices to prove the statement in the special case when $0 \in b(E)$ and $z=0$.

To this end, we use condition (ix) with $k=5, A_{1}=2 E, A_{2}=A_{3}=b(E), A_{4}$ $=\frac{2}{3} b(E), A_{5}=\frac{1}{2} b(E)$ and $p=4$ to find $\Delta \in(0,1)$ having the following property.

Whenever $\delta \in(0, \Delta), I \subset(-\delta, \delta)$ is an open interval with length at least $\delta / 4$ and $\left|\alpha_{i}\right| \leqslant \delta$ for $i=1, \ldots, 5$, then $I \cap \bigcap_{i=1}^{5}\left(\alpha_{i}+A_{i}\right) \notin \mathscr{T}$.

We intend to finish the proof by showing that $f(x)>f(0)-5$ for each $x \in(0, \Delta)$. To prove this, we consider some fixed $x \in(0, \Delta)$ and put

$$
A=(2 E-x) \cap\left(b(E)+\frac{3}{4} x\right) \cap b(E) \cap\left(\frac{2}{3} b(E)+\frac{x}{2}\right) \cap\left(\frac{1}{2} b(E)+\frac{3}{4} x\right) \cap\left(\frac{3}{4} x, x\right) .
$$


We already know that $A \notin \mathscr{T}$. To obtain more useful information, we first denote, for $m=1,2, \ldots$,

$$
E_{m}^{+}=\left\{z \in b(E) ; 0<h<\frac{1}{m} \Rightarrow f(z+h)-f(z-h)>-1\right\},
$$

and

$$
E_{m}^{-}=\left\{z \in b(E) ; 0<h<\frac{1}{m} \Rightarrow f(z-h)-f(z+h)>-1\right\} .
$$

From the symmetric semicontinuity of $f$ at a.e. point of $b(E)$ and (ii) we see that $b(E) \backslash \cup_{m=1}^{\infty}\left(E_{m}^{+} \cup E_{m}^{-}\right) \in \mathscr{T}$. Since (vi), the measurability of $b(E)$ and (viii) imply

$$
A \subset\left(b(E)+\frac{3}{4} x\right) \cap b(E)=b\left(\left(E+\frac{3}{4} x\right) \cap b(E)\right)
$$

and since

$$
\left(E+\frac{3}{4} x\right) \cap b(E) \backslash \bigcup_{m=1}^{\infty}\left[b\left(\left(E+\frac{3}{4} x\right) \cap E_{m}^{+}\right) \cup b\left(\left(E+\frac{3}{4}\right) \cap E_{m}^{-}\right)\right] \in \mathscr{T}
$$

according to (ii), we see that

$$
A \cap \bigcup_{m=1}^{\infty}\left[b\left(\left(E+\frac{3}{4} x\right) \cap E_{m}^{+}\right) \cup b\left(\left(E+\frac{3}{4} x\right) \cap E_{m}^{-}\right)\right] \notin \mathscr{T} .
$$

Thus there is $y \in A$ and a natural number $q$ such that

$$
y \in b\left(\left(E+\frac{3}{4} x\right) \cap E_{q}^{+}\right) \cup b\left(\left(E+\frac{3}{4} x\right) \cap E_{q}^{-}\right) .
$$

Put

$$
C^{+}=\left(E-\frac{3}{4} x+\frac{1}{2} y\right) \cap \bigcup_{m=1}^{\infty} E_{m}^{+}, \quad C^{-}=\left(E-\frac{3}{4} x+\frac{1}{2} y\right) \cap \bigcup_{m=1}^{\infty} E_{m}^{-},
$$

and let $\delta(t)=\min \left(\frac{1}{m}, \frac{x}{4}\right)$ for each $t \in\left(E_{m}^{+} \cup E_{m}^{-}\right) \backslash\left(E_{m-1}^{+} \cup E_{m-1}^{-}\right)$. Denote also

$$
C=\bigcup_{t \in C^{+}}(t-\delta(t), t] \cup \bigcup_{t \in C^{-}}[t, t+\delta(t))
$$

We will show that

$$
2\left(y-\frac{3}{4} x\right) \in b(C) \text {. }
$$

Since $b(E)$ is measurable and $b(E) \backslash \bigcup_{m=1}^{\infty}\left(E_{m}^{+} \cup E_{m}^{-}\right) \in \mathscr{T}$, (i), (viii) and (vi) imply that

$$
b(C) \supset b\left(\left(E-\frac{3}{4} x+\frac{1}{2} y\right) \cap b(E)\right)=\left(b(E)-\frac{3}{4} x+\frac{1}{2} y\right) \cap b(E) .
$$

Thus (*) holds, since $2\left(y-\frac{3}{4} x\right) \in b(E)$ follows from $y \in\left(\frac{1}{2} b(E)+\frac{3}{4} x\right)$ and $2\left(y-\frac{3}{4} x\right) \in\left(b(E)-\frac{3}{4} x+\frac{1}{2} y\right)$ follows from $y \in\left(\frac{2}{3} b(E)+\frac{1}{2} x\right)$.

Since $y \in\left(\frac{3}{4} x, x\right)$, we may find $\beta \in(0, \min (1 / q, x / 4))$ such that $(y-\beta, y+\beta)$ $\subset\left(\frac{3}{4} x, x\right)$. Let $D=\left(E_{q}^{+} \cap(y-\beta, y)\right) \cup\left(E_{q}^{-} \cap(y, y+\beta)\right)$. If

$$
y \in b\left(\left(E+\frac{3}{4} x\right) \cap E_{q}^{+}\right)
$$

then, since $\frac{1}{2} C+\frac{3}{4} x$ is measurable and $y \in b\left(\frac{1}{2} C+\frac{3}{4} x\right)$ (which follows from $(*)$ ), we see from Remark 1(e) that

$$
\left(\frac{1}{2} C+\frac{3}{4} x\right) \cap\left(E+\frac{3}{4} x\right) \cap E_{q}^{+} \cap(y-\beta, y) \neq \varnothing .
$$

Similarly, when $y \in b\left(\left(E+\frac{3}{4} x\right) \cap E_{q}^{-}\right)$, we may prove that

$$
\left(\frac{1}{2} C+\frac{3}{4} x\right) \cap\left(E+\frac{3}{4} x\right) \cap E_{q}^{-} \cap(y, y+\beta) \neq \varnothing .
$$


Hence, in both cases,

$$
\left(\frac{1}{2} C+\frac{3}{4} x\right) \cap\left(E+\frac{3}{4} x\right) \cap D \neq \varnothing,
$$

and we may find $u \in\left(\frac{1}{2} C+\frac{3}{4} x\right) \cap\left(E+\frac{3}{4} x\right) \cap D$. Then $\tau=2\left(u-\frac{3}{4} x\right) \in C$ and consequently $\tau \in(t-\delta(t), t]$ for some $t \in C^{+}$or $\tau \in[t, t+\delta(t))$ for some $t \in C^{-}$. Now we are ready to estimate the difference

$$
\begin{aligned}
f(x)-f(0)= & (f(x)-f(y))+(f(y)-f(2 u-y)) \\
& +(f(2 u-y)-f(2 t-\tau)) \\
& +(f(2 t-\tau)-f(\tau))+(f(\tau)-f(0)) .
\end{aligned}
$$

Since $y \in A \subset(2 E-x) \cap\left(\frac{3}{4} x, x\right)$ there is a $v \in E$ such that $y=2 v-x$ and hence $f(x)-f(y)>-1$.

If $u<y$ then $u \in E_{q}^{+}$(since $u \in D$ ) and, since $y-u<\beta<1 / q$, we get $f(y)-f(2 u-y)>-1$. Similarly, if $u>y$, then $y \in E_{q}^{-}$and $f(y)-f(2 u-y)>$ -1 . To estimate the third term, we note first that, since $\tau=2\left(u-\frac{3}{4} x\right)$,

$$
\frac{1}{2}((2 u-y)+(2 t-\tau))=t+\frac{3}{4} x-\frac{1}{2} y .
$$

Since $t \in C^{+} \cup C^{-} \subset E-\frac{3}{4} x+\frac{1}{2} y$, the center of the rotation is in $E$. Moreover, since $u \in\left(\frac{3}{4} x, x\right),|u-y|<\beta,|t-\tau|<\delta(t) \leqslant \frac{1}{4} x$ and

$$
\begin{aligned}
\frac{1}{2}((2 u-y)-(2 t-\tau)) & =\frac{1}{2}(u-\tau)+\frac{1}{2}(u-y)-(t-\tau) \\
& =\frac{1}{2}\left(\frac{3}{4} x-u\right)+\frac{1}{2}(u-y)-(t-\tau),
\end{aligned}
$$

we see $0<\frac{1}{2}((2 u-y)-(2 t-\tau))<\frac{1}{2} x<1$. Hence $f(2 u-y)-f(2 t-\tau)>-1$. The inequality $f(2 t-\tau)-f(\tau)>-1$ is obvious from the choice of $t$. Finally, $f(\tau)-f(0)>-1$, since $\frac{1}{2} \tau=u-\frac{3}{4} x \in E$ (because $u \in E+\frac{3}{4} x$ ). Summing up, we get $f(x)-f(0)>-5$ and hence the statement of the lemma.

THEOREM 1. Let $f$ be a real-valued function defined on the real line $R$. Then $f$ is continuous at almost every point of the set

$$
R \backslash b(\{x \in R ; \text { fis not symmetrically semicontinuous at } x\}) .
$$

Proof. Let

$$
F=R \backslash b(\{x \in R ; f \text { is not symmetrically semicontinuous at } x\}) .
$$

For each natural $n$ and $k$ we denote

$$
E_{n, k}^{+}=\{x \in F ; 0<h<1 / n \Rightarrow f(x+h)-f(x-h)>-1 / k\}
$$

and

$$
E_{n, k}^{-}=\{x \in F ; 0<h<1 / n \Rightarrow f(x-h)-f(x+h)>-1 / k\} .
$$

From (i) we see that

$$
\bigcup_{n, k}\left(b\left(E_{n, k}^{+}\right) \cup b\left(E_{n, k}^{-}\right)\right) \subset b(F)
$$

and therefore, since $F$ is measurable, $f$ is symmetrically semicontinuous at a.e. point of $\cup_{n, k}\left(b\left(E_{n, k}^{+}\right) \cup b\left(E_{n, k}^{-}\right)\right)$. 
For each pair $n$ and $k$ of natural numbers we use Lemma 1 twice, first, to the function $f$ and the set $E_{n, k}^{+}$and, second, to the function $-f(-t)$ and the set $-E_{n, k}^{+}$. In this way we prove that

$$
-5 / k+\limsup _{x \rightarrow z_{-}} f(x) \leqslant f(z) \leqslant \liminf _{x \rightarrow z_{+}} f(x)+5 / k
$$

for every $z \in b\left(E_{n, k}^{+}\right)$. Hence

$$
\limsup _{x \rightarrow z_{-}} f(x) \leqslant f(z) \leqslant \liminf _{x \rightarrow z_{+}} f(x)
$$

for every $z \in \bigcap_{k=1}^{\infty} \bigcup_{n=1}^{\infty} b\left(E_{n, k}^{+}\right)$.

Since the set of all points $z \in R$ at which either

$$
\limsup _{x \rightarrow z_{-}} f(x) \neq \limsup _{x \rightarrow z_{+}} f(x), \text { or } \liminf _{x \rightarrow z_{-}} f(x) \neq \liminf _{x \rightarrow z_{+}} f(x)
$$

is countable (see, e.g., Young [10]) and since countable sets belong to $\mathscr{T}, f$ is continuous at a.e. point of $\bigcap_{k=1}^{\infty} \cup_{n=1}^{\infty} b\left(E_{n, k}^{+}\right)$. Similarly, applying Lemma 1 to the function $-f$ and the set $E_{n, k}^{-}$and to the function $f(-t)$ and the set $-E_{n, k}^{-}$and again using Young's theorem, we see that $f$ is continuous at almost every point of the set $\bigcap_{k=1}^{\infty} \cup_{n=1}^{\infty} b\left(E_{n, k}^{-}\right)$.

Finally, since $F \backslash \bigcup_{n=1}^{\infty}\left(b\left(E_{n, k}^{+}\right) \cup b\left(E_{n, k}^{-}\right)\right) \in \mathscr{T}$ for every $k$,

$$
F \backslash \bigcap_{k=1}^{\infty}\left(\bigcup_{n=1}^{\infty}\left(b\left(E_{n, k}^{+}\right) \cup b\left(E_{n, k}^{-}\right)\right)\right) \in \mathscr{T} \text {. }
$$

Consequently, $f$ is continuous at a.e. point of $F$.

The next two corollaries follow immediately from Theorem 1. The first, concerning Lebesgue measure, has recently been proved by Belna [1] and the second was proved in 1937 by Fried [3].

COROLlaRY 1. If a function $f$ is symmetrically continuous at almost every point of a measurable set $E \subset R$, then $f$ is continuous almost everywhere in $E$.

COROLlaRY 2. If a function $f$ is symmetrically continuous on a set residual in $R$, then $f$ is continuous on a set residual in $R$.

Theorem 1 also gives us a new way to deduce the results of [9]. In the proofs of these results, we make use of the fact that the existence of the symmetric derivative implies symmetric semicontinuity. An immediate consequence of this is

COROLlaRY 3. If a function $f$ has a symmetric derivative almost everywhere in $R$, then $f$ is measurable.

Now it is also very easy to prove the main lemma from [9] for measure as well as for category.

Lemma 2. Let $f$ be a real-valued function defined on the real line, $K \in R$ and let $E=b\left(\left\{z \in R ; \underline{f}^{s}(z)>K\right\}\right) \backslash b(\{z \in R ; f$ is not symmetrically semicontinuous at $x\})$. Then $\underline{D} f(z) \geqslant K$ at almost every point $z \in E$. 
Proof. From Theorem 1 we see that the function $f$ is continuous almost everywhere in $E$. Let us denote

$$
E_{n}=\left\{z \in E ; 0<h<\frac{1}{n} \Rightarrow \frac{f(z+h)-f(z-h)}{2 h}>K\right\}
$$

and let $z \in b\left(E_{n}\right)$ be a point of continuity of $f$. We can assume $z=0$. If we put in (ix) $A_{1}=2 b\left(E_{n}\right), A_{2}=2 E_{n}, \alpha_{1}=0, \alpha_{2}=-x$, and $p=2$ we find a $\Delta \in(0,1 / n)$ such that for each $x \in(0, \Delta)$ the set $2 b\left(E_{n}\right) \cap\left(2 E_{n}-x\right) \cap(0, x)$ does not belong to $\mathscr{T}$. Hence there are $u \in b\left(E_{n}\right) \cap(0, x / 2)$ and $v \in E_{n} \cap(0, x)$ for which $2 u=2 v$ $-x$ and therefore $f(x)-f(2 u)>(x-2 u) K$. As $u$ lies in $b\left(E_{n}\right)$, there exists a sequence $\left\{t_{i}\right\}_{i=1}^{\infty} \subset E_{n} \cap(u, 2 u)$ with $\lim _{i \rightarrow \infty} t_{i}=u$. From $0<2 u-t_{i}<1 / n$ and $t_{i} \in E_{n}$ it follows that

$$
f(2 u)-f\left(2 t_{i}-2 u\right)>2\left(2 u-t_{i}\right) K
$$

and hence

$$
f(x)-f\left(2 t_{i}-2 u\right)>\left(x+2\left(u-t_{i}\right)\right) K .
$$

Since $\lim _{i \rightarrow \infty} t_{i}=u$ and since $f$ is continuous at 0 , we immediately obtain

$$
f(x)-f(0)=\lim _{i \rightarrow \infty}\left(f(x)-f\left(2 t_{i}-2 u\right)\right) \geqslant x K .
$$

This inequality holds for each $x \in(0, \Delta)$, hence $\underline{D}^{+} f(0) \geqslant K$. Using the above result for the function $-f(-t)$, we prove that $\underline{D}^{-} f(0) \geqslant K$ and thus $\underline{D} f(0) \geqslant K$. Since $E \backslash \bigcup_{n=1}^{\infty} E_{n} \in \mathscr{T}$ and hence $E \backslash \bigcup_{n=1}^{\infty} b\left(E_{n}\right) \in \mathscr{T}$, and since $f$ is continuous a.e. in $E, \underline{D} f(z) \geqslant K$ at almost every point $z \in E$. This completes the proof of the lemma.

The next theorem (proved in [9]) is a generalisation of Khintchine's theorem from [4]. In its formulation we consider the case of sets of Lebesgue measure zero. Thus $b(A)$ denotes the set of all outer density points of $A$.

THEOREM 2. Let $f$ be a real-valued function defined on the real line. Let $E$ be a measurable subset of

$$
b\left(\left\{x \in R ; \bar{f}^{s}(x)<+\infty \text { or } \underline{f}^{s}(x)>-\infty\right\}\right)
$$

at almost every point of which $f$ is symmetrically semicontinuous. Then $f$ is differentiable at almost every point of $E$.

Proof. Let $E_{n}^{+}=\left\{x \in R ; \underline{f}^{s}(x)>-n\right\}, E_{n}^{-}=\left\{x \in R ; \bar{f}^{s}(x)<n\right\}$ and $A=$ $\{x \in R ; f$ is not symmetrically semicontinuous at $x\}$. Using the preceding lemma, we see that $\underline{D} f>-\infty$ at almost every point of each of the sets $b\left(E_{n}^{+}\right) \backslash b(A)$, and that $\bar{D} f<+\infty$ at almost every point of each of the sets $b\left(E_{n}^{-}\right) \backslash b(A)$. From [7, p. 171, Theorem 3] we deduce that $f$ is differentiable at almost every point of $\cup_{n=1}^{\infty}\left(b\left(E_{n}^{+}\right) \cup b\left(E_{n}^{-}\right)\right) \backslash b(A)$. Hence it is differentiable at almost every point of the set $b\left(\cup_{n=1}^{\infty}\left(E_{n}^{+} \cup E_{n}^{-}\right)\right) \backslash b(A)$, which completes the proof.

Corollary 4 follows immediately from Theorem 2 , or it is also a consequence of Corollary 3 and Khintchine's theorem mentioned above.

COROLlaRY 4. If a function $f$ is symmetrically differentiable almost everywhere in $R$, then $f$ is differentiable almost everywhere in $R$. 
In the case of category, we deduce a similar result from Lemma 1 . In its formulation $b(A)$ means the set $R \backslash \operatorname{Closure}(a(A))$.

THEOREM 3. If $f$ is a real-valued function defined on the real line, then $f$ is differentiable on a set residual in the open set $E=b\left(\left\{x \in R ; f^{s}(x)\right.\right.$ exists $\left.\}\right) \backslash$ Closure $(b(\{x \in R ; f$ is not symmetrically semicontinuous at $x\}))$.

Proof. Let $R_{1}$ ( $R_{1}^{+}$, respectively) denote the set of all rational numbers (positive rational numbers, respectively). For each $r \in R_{1}$ and each $s \in R_{1}^{+}$we denote

$$
\begin{aligned}
& B(s)=\left\{x \in E ; \underline{f}^{s}(x)>s\right\}, \\
& B(-s)=\left\{x \in E ; \bar{f}^{s}(x)<-s\right\}, \text { and } \\
& B(r, s)=\left\{x \in E ; r<\underline{f}^{s}(x) \leqslant \bar{f}^{s}(x)<r+s\right\} .
\end{aligned}
$$

Using Lemma 2 we see that

$$
\begin{aligned}
& b(B(s)) \backslash\{x \in E ; \underline{D} f(x) \geqslant s\} \in \mathscr{T}, \\
& b(B(-s)) \backslash\{x \in E ; \bar{D} f(x) \leqslant-s\} \in \mathscr{T}, \\
& b(B(r, s)) \backslash\{x \in E ; r \leqslant \underline{D} f(x) \leqslant \bar{D} f(x) \leqslant r+s\} \in \mathscr{T},
\end{aligned}
$$

and using (vi) also that

$$
b\left(\bigcup_{r \in R_{1}} B(r, s)\right) \backslash\{x \in E ; \bar{D} f(x)-\underline{D} f(x) \leqslant s\} \in \mathscr{T} .
$$

Evidently

$$
C=\bigcap_{s \in R_{1}^{+}} B(s) \cup \bigcap_{s \in R_{1}^{+}} B(-s) \cup \bigcap_{s \in R_{1}^{+}} \bigcup_{r \in R_{1}} B(r, s) \supset\left\{x \in E ; f^{s}(x) \text { exists }\right\}
$$

and hence $b(C) \supset E$. Using (vi) once again we obtain

$$
b(C) \backslash\left(b\left(\bigcap_{s \in R_{1}^{+}} B(s)\right) \cup b\left(\bigcap_{s \in R_{1}^{+}} B(-s)\right) \cup b\left(\bigcap_{s \in R_{1}^{+}} \bigcup_{r \in R_{1}} B(r, s)\right)\right) \in \mathscr{T} .
$$

From the preceding relations we see that

$$
\begin{aligned}
b\left(\bigcap_{s \in R_{1}^{+}} B(s)\right) \backslash\left\{x \in E ; f^{\prime}(x)\right. & =+\infty\} \\
= & b\left(\bigcap_{s \in R_{1}^{+}} B(s)\right) \backslash \bigcap_{s \in R_{1}^{+}}\{x \in E ; \underline{D} f(x) \geqslant s\} \\
= & \bigcup_{p \in R_{1}^{+}}\left(b\left(\bigcap_{s \in R_{1}^{+}} B(s)\right) \backslash\{x \in E ; \underline{D} f(x) \geqslant p\}\right)
\end{aligned}
$$

belongs to $\mathscr{T}$. In the same way we deduce that

$$
b\left(\bigcap_{s \in R_{1}^{+}} B(-s)\right) \backslash\left\{x \in E ; f^{\prime}(x)=-\infty\right\} \in \mathscr{T}
$$


and

$$
b\left(\bigcap_{s \in R_{1}^{+}} \bigcup_{r \in R_{1}} B(r, s)\right) \backslash\left\{x \in E ; f^{\prime}(x) \text { exists and is finite }\right\} \in \mathscr{T} .
$$

These facts immediately imply that $E \backslash\left\{x \in E ; f^{\prime}(x)\right.$ exists $\}$ belongs to $\mathscr{T}$, which completes the proof of Theorem 3 .

We note that some measurability assumptions in our results are necessary. For the case of measure, the corresponding example was given first by Ponomarev [5] and in the case of category another one was mentioned by Belna [1]. The following example, which includes the case of measure as well as the case of category, was communicated to us by D. Preiss.

ExAMPLE. Let $R_{1}$ denote the set of all rational numbers and let $R_{1}(A)$ (for $A \subset R$ ) denote the set of all finite linear combinations of elements of $A$ with rational coefficients.

Let $\gamma$ be the first ordinal having cardinality $2^{\aleph_{0}}$ and let $\left\{F_{\alpha}, \alpha<\gamma\right\}$ be the family of all uncountable Borel subsets of $R$. By transfinite induction we shall define a sequence $\left\{x_{\alpha}, \alpha<\gamma\right\}$ of points of $R$. Let $x_{0}$ be an arbitrary irrational element of $F_{0}$. Whenever $\left\{x_{\alpha}, \alpha<\beta\right\}$ have been defined and $R_{1} \cap R_{1}\left(\left\{x_{\alpha}, \alpha<\beta\right\}\right)=\varnothing$, we note that

$$
\operatorname{card}\left(R_{1}\left(R_{1} \cup\left\{x_{\alpha}, \alpha<\beta\right\}\right)\right) \leqslant \max \left(\boldsymbol{\aleph}_{0}, \operatorname{card} \beta\right)<2^{\aleph_{0}}=\operatorname{card} F_{\beta} .
$$

Hence it is possible to choose $x_{\beta} \in F_{\beta}$ which does not belong to

$$
R_{1}\left(R_{1} \cup\left\{x_{\alpha}, \alpha<\beta\right\}\right) \text {; i.e. } R_{1} \cap R_{1}\left(\left\{x_{\alpha}, \alpha \leqslant \beta\right\}\right)=\varnothing .
$$

Having thus constructed the sequence $\left\{x_{\alpha}, \alpha<\gamma\right\}$, we put $H=R_{1}\left(\left\{x_{\alpha}, \alpha<\gamma\right\}\right)$ and note that $H \cap R_{1}=\varnothing$, every uncountable Borel subset of $R$ meets $H$ and $H$ is symmetric in the sense that $x \in H$ and $x+h \in H$ imply $x-h \in H$. It follows that the characteristic function of $H$ is symmetrically continuous (and even symmetrically differentiable) at every point of $H$, but it is discontinuous everywhere. Moreover, $H$ is full in the sense of measure as well as in the sense of category since every Borel subset of $R \backslash H$ is countable.

ACKnowledgement. The author would like to express his thanks to Professor D. Preiss for his help and constant encouragement.

\section{REFERENCES}

1. C. L. Belna, Symmetric continuity of real functions, Proc. Amer. Math. Soc. 87 (1983), 99-102.

2. Z. Charzynski, Sur les fonctions dont la derivée symetrique est partout finie, Fund. Math. 21 (1933), 214-225.

3. H. Fried, Über die symmetrische Stetigkeit von Funktionen, Fund. Math. 29 (1937), 134-137.

4. A. Khintchine, Recherches sur la structure des fonctions mesurables, Fund. Math. 9 (1927), 212-279.

5. S. P. Ponomarev, O simetričeski něpreryvnych funkcijach, Mat. Zametki 1 (1967), 385-390.

6. D. Preiss, A note on symmetrically continuous functions, Časopis Pěst. Mat. 96 (1971), 262-264.

7. S. Saks, Théorie de l'intégrale, PWN, Warsaw, 1933.

8. E. M. Stein and A. Zygmund, On the differentiability of functions, Studia Math. 23 (1964), 247-283.

9. J. Uher, Symmetrically differentiable functions are differentiable almost everywhere, Real Anal. Exchange 8 (1982-83), 253-261.

10. W. H. Young, La symétrie de structure des fonctions de variables réelles, Bull. Sci. Math. 52 (1928), 265-280.

Leninova 56, 16000 Praha 6, Czechoslovakia 